\title{
Liderazgo y cultura en carreras de ingeniería: estudio en una universidad chilena
}

\author{
Liliana M. Pedraja-Rejas ${ }^{{ }^{\star}}$, Emilio R. Rodríguez-Ponce ${ }^{2}$, Constanza J. Espinoza-Marchant ${ }^{1}$ y Camila P. Muñoz-Fritis ${ }^{1}$ \\ (1) Ingeniería Industrial y de Sistemas, Universidad de Tarapacá, 18 de septiembre 2222, Arica- Chile. \\ (correo-e: Ipedraja@uta.cl: conniee.marchant@gmail.com: camila.munoz.fritis@gmail.com) \\ (2) Instituto de Alta Investigación, Universidad de Tarapacá, Antofagasta 1520, Arica-Chile. \\ (correo-e: erodriguez@uta.cl)
}

* Autor a quien debe ser dirigida la correspondencia.

Recibido May. 5, 2020; Aceptado Jul.17, 2020; Versión final Ago. 27, 2020, Publicado Feb. 2021

\begin{abstract}
Resumen
El objetivo de esta investigación es identificar la percepción que tienen los docentes de las carreras de ingeniería sobre el estilo de liderazgo de sus directivos y el tipo de cultura organizacional presente en sus respectivas unidades académicas. Se lleva a cabo un análisis descriptivo de las percepciones de 35 docentes de una universidad chilena, recopiladas mediante la aplicación de un cuestionario basado en los modelos de Bass y Avolio (MLQ) y Cameron y Quinn (OCAI). Los hallazgos indican que los directivos de ingeniería en esta institución no poseen un liderazgo definido, sino más bien presentan conductas ocasionales de los tres estilos (transformacional, transaccional y pasivo/evitador). La cultura de mercado, enfocada en los resultados académicos, es predominante por sobre la cultura jerárquica, de clan y adhocrática. Se concluye que este estudio abre la oportunidad de llevar la investigación del liderazgo y la cultura organizacional a niveles de facultades y departamentos, ámbitos que son poco explorados aún en la educación superior.
\end{abstract}

Palabras clave: liderazgo; cultura organizacional; educación superior; formación educativa; ingeniería

\section{Leadership and culture in engineering programs: study at a Chilean university}

\begin{abstract}
The aim of this research study is to assess engineering faculty perception of their deans' leadership styles and of the organizational culture present at their faculties. The perceptions of 35 academics from one Chilean university were compiled by applying a questionnaire based on the Bass and Avolio (MLQ) model and the Cameron and Quinn (OCAI) model. A descriptive analysis was performed. The results showed that engineering deans do not have a defined leadership, but showed occasional behaviors of three different styles (transformational, transactional, and passive/avoidant). Market culture, focused on academic results, was predominant over hierarchical, clan, and adhocracy culture. It is concluded that the present study opens the opportunity to conduct leadership and organizational culture research at the faculty and department levels, which still remains largely unexplored in higher education.
\end{abstract}

Keywords: leadership; organizational culture; higher education; educational training; engineering 


\section{INTRODUCCIÓN}

En la actualidad, las instituciones de educación superior se enfrentan a grandes desafíos, entre ellos, la globalización, las estructuras de financiación, y la oferta y demanda cada vez más cambiantes, es por esto que, en la lucha por la supervivencia, están en constante búsqueda de ventajas competitivas (Elrehail et al., 2018). En este sentido, la gestión de conocimiento se considera como una de las principales fuentes de esta y se realza su importancia como factor fundamental para la sostenibilidad y el éxito a largo plazo de la institución (Lee, 2018). Las universidades conscientes de esto, se han preocupado y ocupado de los altos niveles de competitividad en la industria educativa, y es por esto que han dado gran relevancia a la excelencia, la cual está estrechamente relacionada con los conceptos de calidad e innovación (Sainz-Martín et al., 2017). Esto ha conllevado a su vez al rediseño de mallas curriculares centradas no solo en conocimientos técnicos sino además en otro tipo de habilidades acorde a la dinámica del cambio, sentando las bases para el logro de una ventaja competitiva que permita el progreso y el desarrollo de las naciones al crear y difundir conocimiento avanzado (Villa-Peralta, 2017).

En este contexto, el aprendizaje en la educación se debe centrar en la sostenibilidad y en el logro de la calidad, el cual requiere entre otros factores, el compromiso de los académicos, quienes con su motivación e ideas innovadoras pueden materializar el cambio en el contenido y los métodos de enseñanza (Leal Filho et al., 2018), logrando así que los estudiantes sean capaces de aprovechar y aplicar el conocimiento aprendido, además de pensar de manera cuidadosa, crítica y creativa (Cawn et al., 2016). En esta línea de pensamiento, los docentes son considerados un punto primordial en el proceso de la educación, ya que estos son los que se relacionan directamente con los alumnos, y son quienes pueden cultivar un sentido de pertenencia en las aulas, lo que está fuertemente relacionado con la forma en que los estudiantes de las carreras de ciencia e ingeniería se sienten, con cuánto se esfuerzan, y qué tan dispuestos están a participar en una clase (Wilson et al., 2015).

A su turno, la literatura existente sostiene que los programas de desarrollo profesional impartidos por las distintas universidades se ven influenciados por una serie de factores, entre los que se incluyen el contexto, el liderazgo ejercido y la cultura que se promueve en estos lugares (Englund et al., 2018). En efecto, el liderazgo en las instituciones educacionales es considerado de vital importancia, ya que líderes efectivos tienen el potencial de influir en la creación de entornos de aprendizaje que conllevan a mejorar los resultados y los procesos de la organización (Daniëls et al., 2019), asimismo, fomentar una cultura basada en la confianza e interacción social permitiría crear un entorno adecuado para la creatividad individual que finalmente contribuya a la innovación y mejore el rendimiento a nivel institucional, permitiendo apoyar la competitividad de los sistemas educativos y mejorar así los procesos de gestión de conocimiento (Lee, 2018). De igual manera, se reconoce que la cultura organizacional refleja principalmente la influencia de sus líderes a través de sus estrategias, prácticas, valores y estilos de liderazgo (Warrick, 2017), por lo que estos se han vuelto importantes al momento de moldear una cultura robusta que sea congruente con la misión de la institución y que asegure el éxito de esta. Por esta razón resulta ideal contar con líderes transformacionales que sean proactivos y que construyan organizaciones académicas que posean un sentido de comunidad, tomando decisiones basadas en hechos y asegurando así resultados efectivos (Desselle et al., 2017).

Por otro lado, se ha planteado que el concepto de excelencia ha adquirido gran relevancia en el ámbito universitario, ya que involucra todos los procesos de la actividad educativa, incluyendo desde la gestión universitaria hasta los procesos de enseñanza y aprendizaje en las aulas, razón por las que estas instituciones reconocen la importancia en difundir el uso de buenas prácticas de enseñanza basado en conceptos de calidad e innovación (Sainz-Martín et al., 2017). Ahora bien, en el ámbito de la ingeniería, las universidades tienen el desafío de convertir a los estudiantes en ingenieros líderes, permitiendo el desarrollo de competencias globales, como la capacidad de ser innovadores, confiables, competentes, carismáticos, con disposición a buscar oportunidades, tomar decisiones y asumir riesgos (Boulais et al., 2015), lo que ha conllevado a la modificación del currículo en algunos casos. En este sentido, se afirma que los académicos ya no pueden centrarse solo en su propia enseñanza y disciplina, sino más bien existe la necesidad de una colaboración interdisciplinaria del equipo para lograr coherencia y calidad en el diseño curricular, lo que se hace difícil sino se cuenta con un liderazgo comprometido y una cultura que fomente la participación y la colaboración entre los miembros (Newell y Bain, 2019). A partir de lo anterior, se pretende identificar la percepción que tienen los docentes de las carreras de ingeniería de una universidad estatal chilena sobre el estilo de liderazgo de sus directivos y el tipo de cultura organizacional presente en sus respectivas unidades académicas. Para esto, se realizará un estudio de tipo exploratorio, cuantitativo y descriptivo a pequeña escala, de modo de contribuir a la literatura en esta materia.

\section{OTROS ANTECEDENTES}

En esta sección, se revisa y analiza conceptos relacionados con liderazgo y la cultura organizacional en el contexto de la educación superior, enfocándose principalmente en el área de la ingeniería. 


\section{Liderazgo en la educación superior}

En términos generales el liderazgo se define como la capacidad de los líderes para modificar el comportamiento de sus subordinados, logrando motivar y empoderar a estos con el fin de aprovechar el máximo de los conocimientos y habilidades de los seguidores en pro de los objetivos organizacionales. Es por esto que el liderazgo demuestra ser un factor determinante en la eficacia organizacional, ya que además de asegurar una estrategia de mayor calidad para el logro de los objetivos (Riquelme-Castañeda y PedrajaRejas, 2019), tiene el potencial de incentivar la innovación en la organización, motivando a los empleados y creando ambientes propicios para el desarrollo de habilidades creativas, lo que conduce a su vez a ventajas competitivas sostenibles (Elrehail et al., 2018).

Para el caso de las universidades y debido a que estas son organizaciones altamente complejas, el ejercicio de un liderazgo efectivo permite, además de motivar e inspirar a otros, articular una visión atractiva y otorgarle posicionamiento y seguridad a la institución, lo que ayudaría a su vez al logro de la excelencia institucional (Ganga-Contreras et al., 2018), es por esto que se reconoce la importancia de ejercer un liderazgo que garantice estrategias flexibles e integrales, destacando la capacidad de escuchar y respetar las ideas de todos los participantes en este proceso (estudiantes, profesores y otros stakeholders), con el fin de anticipar el cambio, estimular el diálogo, la cooperación, el conocimiento, la creatividad (Leal Filho et al., 2018), y conducir así a un clima de aprendizaje efectivo y procedimientos educativos eficaces (Daniëls et al., 2019). Es más, Berestova et al. (2020) sostienen que el liderazgo docente promueve un crecimiento efectivo dentro del contexto educativo, ya que los académicos que se convierten en líderes contribuyen al desarrollo profesional efectivo de sus compañeros docentes, al crear comunidades líderes de especialistas que exploran y mejoran colectivamente las prácticas de enseñanza y aprendizaje, lo que tiene un efecto favorable en la calidad de la educación y el rendimiento de los estudiantes. De igual manera, los directivos que tienen una sólida comprensión de las prácticas efectivas de liderazgo, son gestores de una cultura y estructura orientada en el aprendizaje continuo, basada en las necesidades de los estudiantes y las capacidades de los académicos (Cawn et al., 2016), razón por la que Pedraja-Rejas et al. (2018a) concluyen que desde un punto de vista conceptual, existe un probable vínculo entre los estilos de liderazgo y los tipos de cultura que prevalecen en las instituciones de educación superior.

Finalmente, en el contexto de la ingeniería, el desarrollo de competencias necesarias en esta área no solo se basan en aplicar conocimientos técnicos en contextos vinculados a la profesión, sino más bien se necesita contar con competencias transversales, incluidas la autonomía, el trabajo en equipo, la capacidad de negociación, la comunicación efectiva y el liderazgo, es por esto que la formación de un ingeniero es mucho más exigente ya que es necesario darle sentido e intencionalidad instructiva, con un enfoque de formación integral para que se desarrolle a niveles globales (Villa-Peralta, 2017). En este sentido, el paso de los estudiantes por la universidad es considerada una fase significativa, ya que es aquí donde los futuros líderes adquieren conocimientos, habilidades y las actitudes que hacen posible su crecimiento personal (Schweisfurth et al., 2018), razones que hacen mucho más atractivo el estudio de variables que pueden afectar al proceso formativo de estos, como es el caso del liderazgo.

\section{Estilos de liderazgo según Bass y Avolio}

Existen diversas tipologías de estilos de liderazgo. En particular uno de los modelos más citados en la literatura es el de Bass y Avolio, quienes tipifican tres estilos: el transformacional, transaccional y pasivo/evitador (Araneda-Guirriman et al., 2016). A continuación, se describe cada uno de ellos y sus respectivos factores.

El liderazgo transformacional es considerado como el estilo de liderazgo más efectivo, y se reconoce como la capacidad de los líderes de elevar y ampliar los intereses de sus empleados al cambiar las percepciones, expectativas y motivaciones de estos para trabajar hacia objetivos comunes. Este estilo se compone de los siguientes factores: Influencia Idealizada, aquí los líderes exhiben comportamientos (Conductual) y características (Atribuida) que hacen que los seguidores se identifiquen con ellos, llegando a considerarlos como modelos a seguir; Motivación Inspiracional, definida como la capacidad del líder de motivar a los seguidores al proporcionar significado y desafío al trabajo de estos; Estimulación Intelectual, acá el líder hace que los seguidores cuestionen las formas probadas de resolver problemas, y los alienta a replantearse los métodos que utilizan con el objetivo de mejorarlos, proporcionándoles autonomía y empoderamiento en el trabajo; y por último, Consideración Individualizada, aquí el líder se centra en comprender las necesidades de cada seguidor y trabaja continuamente para que desarrollen todo su potencial, asimismo el líder se caracteriza por apoyarlos, convirtiéndose así en un mentor para ellos (Araneda-Guirriman et al., 2016; Bass et al., 2003; Elrehail et al., 2018). 
Por otro lado, el liderazgo transaccional se enfoca principalmente en promover el cumplimiento de los seguidores a través de recompensas y castigos. Este se compone de dos factores, los cuales son: Recompensa Contingente, aquí el líder aclara las expectativas y lo que recibirán los seguidores si cumplen con los niveles esperados de rendimiento; y Administración por Excepción Activa, la cual ocurre cuando el líder se enfoca en monitorear la ejecución de las tareas para detectar de manera anticipada el surgimiento de problemas, y no afectar así los niveles de rendimiento deseados (Bass et al., 2003; Lai et al., 2018).

Finalmente, el estilo pasivo/evitador se caracteriza porque el líder no motiva ni guía a los seguidores, además retrasa las decisiones y evita intervenir hasta que algo sale mal. Este estilo se compone por las dimensiones: Laissez-faire, aquí el líder evita aclarar expectativas, proporcionar objetivos y estándares, interactuar en asuntos críticos y delega la autoridad a otros; y Administración por Excepción Pasiva, la cual ocurre cuando el líder espera a que surjan problemas antes de tomar alguna medida, y tiende a intervenir con críticas al involucrarse después de que los subordinados cometen errores o no cumplen con las expectativas (Bass et al., 2003; Lai et al., 2018).

\section{Cultura organizacional}

En términos generales, la cultura organizacional se considera como el conjunto de prácticas, símbolos, valores, creencias y suposiciones implícitas que los miembros de una institución comparten (Bortolotti et al., 2015). Es por esto que la cultura es considerada como un concepto complejo, y que se determina por la capacidad de movilizar todos los recursos disponibles con el fin de lograr los objetivos estratégicos y el cumplimiento de la misión, permitiendo así la satisfacción de todas las partes interesadas (internas y externas) (Boichuk y Fast, 2017). Warrick (2017) igualmente sostiene que la cultura podría generar diversos efectos en las organizaciones, ya que podría promover un excelente ambiente de trabajo lo que conllevaría a su vez a obtener muy buenos resultados, pero por otro lado podría generar ambientes de trabajo disfuncionales llenos de estrés y tensión.

En relación a la cultura organizacional en el ámbito de la educación superior, Vasyakin et al. (2016) sostienen que esta permite el tipo de reflexividad necesaria para que los administradores clarifiquen la identidad de las instituciones mientras que destacan sus cualidades únicas. En este sentido, estudiar la cultura organizacional en universidades es un caso bastante especial, debido a que estos son lugares que se basan en los principios del conocimiento y el aprendizaje. Es más, Vasyakin et al. (2016), plantean que la cultura en estos lugares se debería orientar a la generación de competencias esenciales, la armonización de las relaciones internas, la realización personal y profesional de docentes y alumnos, y a la explotación del potencial creativo.

Por su parte, un factor a considerar es que las universidades están formadas por diversos departamentos, escuelas y facultades que funcionan como subsistemas de un todo, las cuales a pesar de que se puedan identificar con diferentes tipos de culturas, ya sea en sus formas de enseñar, aprender e investigar, la cultura organizacional de la universidad igual influiría sobre cada una de estas pequeñas organizaciones. En este contexto, existen investigaciones que indican que los estudiantes de carreras de ingeniería se verían afectados por la cultura organizacional compartida por la institución durante su proceso formativo, especialmente en características importantes como la capacidad para trabajar en equipo (Rodzalan y Saat, 2013). Es por esto que se reconoce que para lograr el cambio cultural se requiere una transformación, formar nuevos conceptos colectivos y crear nuevas creencias sobre lo que se valora en la educación de la ingeniería (Carberry y Baker, 2018). De aquí nace la necesidad de planificar una gama de iniciativas destinadas a mejorar los componentes que ayudan a apoyar y desarrollar la cultura organizacional dentro de las distintas unidades, y se considera que la promoción de la innovación, la adhesión a las prácticas comerciales y la orientación a los resultados deberían contribuir de manera adecuada al crecimiento futuro de estas áreas y de la universidad en general (Vasyakin et al., 2016).

Finalmente, la importancia de conocer la cultura organizacional radica primeramente en que esta permite crear unidades académicas efectivas, productivas e innovadoras (Caliskan y Zhu, 2019), y segundo, que a pesar de que esta es única y distintiva para cada una de las unidades, no implica bajo ninguna circunstancia que no sea dinámica y que no pueda adaptarse a los nuevos requerimientos (Chidambaranathan y Rani, 2015; Pedraja-Rejas et al., 2018b). Por esto es que se hace necesario el diseño y la implementación de un modelo de diagnóstico cultural que identifique los elementos primordiales para favorecer la puesta en marcha de una estrategia de restructuración y organización, y que se encuentre en correspondencia con el quehacer académico llevado adelante por la unidad (González et al., 2016).

\section{Tipos de cultura organizacional según Cameron y Quinn}

Cameron y Quinn (2006) plantean un modelo que distingue dos dimensiones, uno corresponde al continuo entre flexibilidad contra estabilidad, mientras que el otro eje se articula entre procesos internos frente al 
posicionamiento externo, de esta manera surgen las culturas de clan, adhocrática, de mercado y jerárquica. En la Figura 1 se presentan los cuatro tipos de cultura con sus respectivas características.

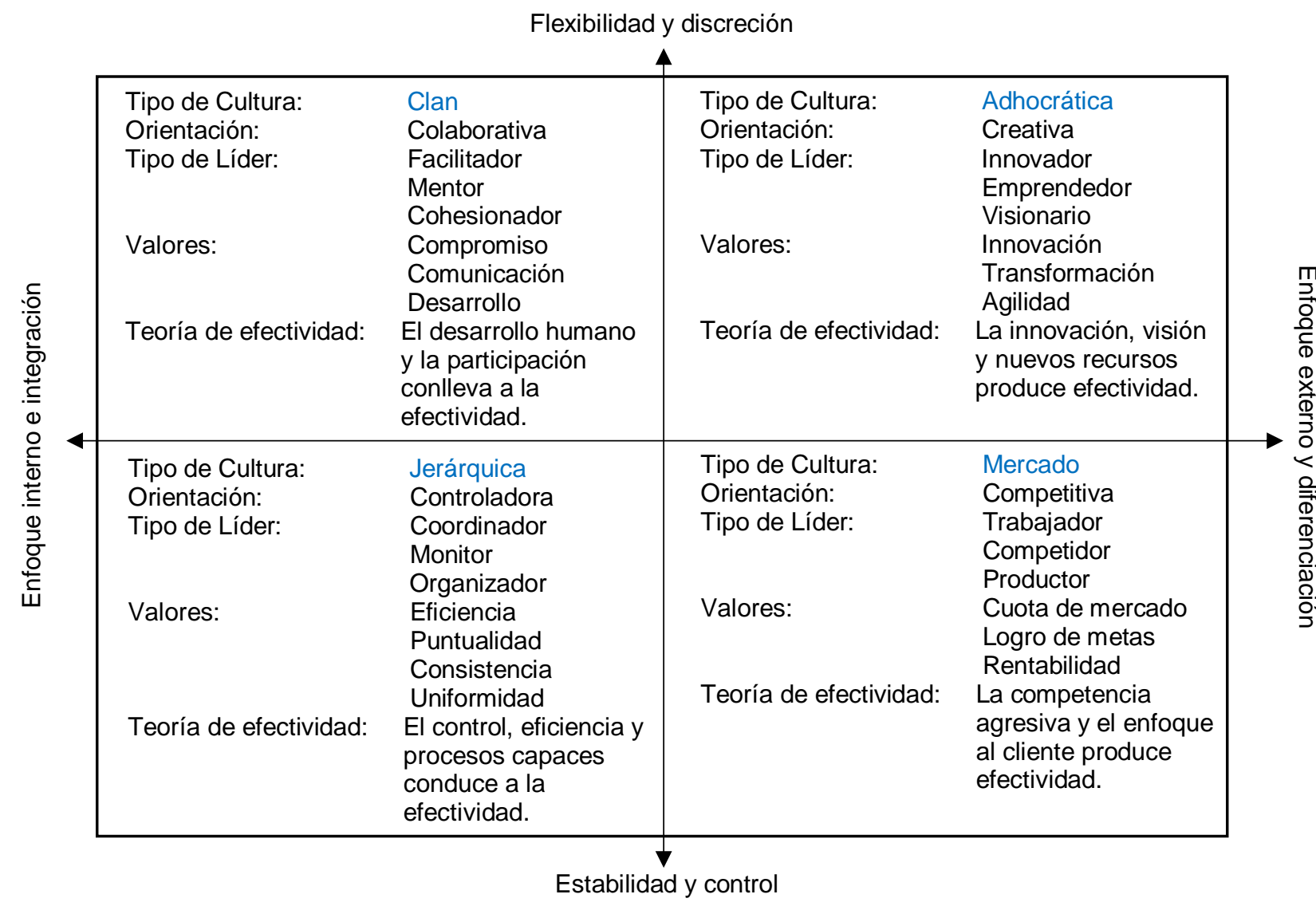

Fig. 1: Modelo de Valores en Competencia (Cameron y Quinn, 2006)

En definitiva, la cultura de clan corresponde al cuadrante de alta flexibilidad y enfoque interno, y le otorga alto énfasis al trabajo en equipo así como a la satisfacción, compromiso, participación y desarrollo de los empleados. En segundo lugar, se destaca la cultura adhocrática, la cual se ubica en el cuadrante de alta flexibilidad pero con un enfoque externo, este tipo de organizaciones enfatizan la creatividad, la innovación, la toma de riesgos y el trabajo dinámico para enfrentar los nuevos desafíos. Por otro lado, la cultura de mercado enfatiza un enfoque externo y de control, de tal forma que se caracteriza por darle importancia a la productividad, eficiencia y racionalidad para cumplir el logro de las metas institucionales. Finalmente, la cultura jerárquica se encuentra en el cuadrante de enfoque interno y alto control, por lo que son organizaciones que valoran la eficiencia, la puntualidad y la previsibilidad, confiando así en las reglas formales y políticas estructuradas para regular el comportamiento de los empleados (Cameron y Quinn, 2006, 2011).

\section{METODOLOGÍA}

El diseño de la investigación fue de carácter: 1) cuantitativo, ya que se hizo uso de instrumentos para conocer las percepciones de los participantes; 2) de corte transversal, debido a que captura las impresiones de una temática en un momento específico del tiempo; 3) descriptivo, ya que se busca identificar y describir las características en relación a las variables en estudio; y 4) con un propósito exploratorio, debido a que la materia no ha sido suficientemente abordada en el contexto de la ingeniería. Igualmente en este estudio se trabaja a pequeña escala, lo que para Fosnacht et al. (2017) resulta pertinente, ya que estos autores sostienen que un esfuerzo adicional para aumentar las tasas de respuesta de los cuestionarios con frecuencia solo cambian los resultados de manera trivial. De esta manera la investigación permite "identificar mediante datos empíricos aspectos fundamentales que den una visión general, de tipo aproximativo" (Pedraja-Rejas et al., 2018b, p. 732) de las percepciones de los docentes de ingeniería de una universidad chilena sobre sus líderes, así como la cultura en sus respectivas unidades académicas.

\section{Participantes y recolección de información}

Los participantes del estudio fueron elegidos de manera no probabilística y no representativa, utilizando un método de muestreo por conveniencia debido a que el proceso de la encuesta dependía únicamente de la 
disponibilidad con la que contaban los académicos. Los requisitos que los docentes debían cumplir para participar eran: 1) pertenecer a cualquiera de las unidades académicas de ingeniería de la universidad estudiada; 2) tener una modalidad contractual de jornada completa o media jornada; y 3) no ejercer como líderes formales en ninguna unidad académica.

En definitiva, se obtuvo la participación de 35 docentes, lo que corresponde al $70 \%$ del universo total. De esta muestra, el $91,4 \%$ son hombres y solo el $8,6 \%$ mujeres. De igual manera, el $80 \%$ de estos pertenecen a la dotación de planta, mientras que el $20 \%$ a contrata. Por último, en relación al grado académico máximo de los participantes, el $40 \%$ de estos tiene un doctorado, el $51,4 \%$ algún máster y solo el $8,6 \%$ posee únicamente el título profesional.

\section{Variables, dimensiones y medidas del cuestionario}

El liderazgo considera las variables: transaccional, transformacional y pasivo/evitador. Para medir esto, se adaptó el modelo MLQ (Multifactor Leadership Questionnaire) de los autores Bass y Avolio (2000) y se utilizó una escala tipo Likert de 7 puntos. Este cuestionario fue sometido primeramente a una prueba piloto, y en definitiva quedó compuesto de 49 preguntas. En la Tabla 1 se muestra el número de ítems por cada dimensión y sus respectivos niveles de confiabilidad.

Tabla 1: Confiabilidad de los estilos de liderazgo y sus dimensiones

\begin{tabular}{|l|l|l|l|}
\hline Estilo de Liderazgo & \multicolumn{1}{|c|}{ Dimensiones } & \multicolumn{2}{c|}{ Alfa de Cronbach } \\
\hline \multirow{2}{*}{$\begin{array}{l}\text { Liderazgo } \\
\text { transaccional }\end{array}$} & Recompensa contingente (4 ítems) & $\alpha=0,811$ & \multirow{2}{*}{$\alpha=0,892$} \\
\cline { 2 - 3 } & Dirección por excepción activa (4 ítems) & $\alpha=0,867$ & \\
\hline \multirow{4}{*}{$\begin{array}{l}\text { Liderazgo } \\
\text { transformacional }\end{array}$} & Influencia idealizada conductual (6 ítems) & $\alpha=0,940$ & \\
\cline { 2 - 3 } & Influencia idealizada atribuida (5 ítems) & $\alpha=0,946$ & \multirow{2}{*}{$\alpha=0,987$} \\
\cline { 2 - 3 } & Motivación inspiracional (6 ítems) & $\alpha=0,955$ \\
\cline { 2 - 3 } & Estimulación intelectual (6 ítems) & $\alpha=0,914$ & \\
\cline { 2 - 3 } & Consideración individualizada (6 ítems) & $\alpha=0,950$ & \\
\hline \multirow{2}{*}{ Pasivo/evitador } & Laissez-faire (8 ítems) & $\alpha=0,898$ & \multirow{2}{*}{$\alpha=0,903$} \\
\cline { 2 - 3 } & Dirección por excepción pasiva (4 ítems) & $\alpha=0,769$ & \\
\hline
\end{tabular}

Por otro lado, la cultura organizacional considera las variables: clan, adhocrática, de mercado y jerárquica. Para medir este ítem se adaptó el Instrumento de Valoración de Cultura Organizacional (OCAI) diseñado por Cameron y Quinn (2011), el cual está basado en el Modelo de Valores en Competencia (MVC) de los mismos autores. Este instrumento cuenta con 6 dimensiones, las cuales son: características dominantes, liderazgo organizacional, gestión de empleados, cohesión organizacional, énfasis estratégico y criterios de éxito. Por último, y al igual que el cuestionario anterior, se utilizó una escala Likert de 1 a 7 , y se sometió a una prueba piloto para analizar la confiabilidad del instrumento. En la Tabla 2 se observa el número de preguntas por cada tipo de cultura y sus respectivos niveles de confiabilidad.

Tabla 2: Confiabilidad por tipo de cultura

\begin{tabular}{|l|c|c|}
\hline \multicolumn{1}{|c|}{ Tipo de Cultura } & Total de preguntas & Alfa de Cronbach \\
\hline Cultura de clan & 6 ítems & $\alpha=0,932$ \\
\hline Cultura adhocrática & 6 ítems & $\alpha=0,896$ \\
\hline Cultura de mercado & 6 ítems & $\alpha=0,906$ \\
\hline Cultura jerárquica & 6 ítems & $\alpha=0,868$ \\
\hline
\end{tabular}

Por último, la confiabilidad (medido por el alfa de Cronbach) del cuestionario utilizado presenta índices adecuados, ya que todos son mayores a 0,7 , el cual es el valor mínimo aceptado para considerar como fiable el instrumento (Taber, 2017).

\section{Análisis de Datos}

Para el procesamiento de los datos del liderazgo se utilizó el software IBM SPSS versión 24.0, el cual permitió la obtención de la estadística descriptiva para cada estilo. Luego se calcularon los porcentajes de cada respuesta posible ( 1 al 7) de cada dimensión. Por otro lado, en el análisis de la cultura se calculó el promedio 
obtenido de las respuestas de los académicos, las cuales fueron llevadas posteriormente a porcentajes relativizados por el máximo puntaje posible alcanzado (7) y se realizó el gráfico radial correspondiente. De esta manera fue posible efectuar las comparaciones para conocer e interpretar el factor más valorado de cada una de las variables.

\section{RESULTADOS}

En la Tabla 3 se muestran los estadísticos descriptivos sobre el liderazgo de los directivos de ingeniería. Para el caso del estilo transaccional se obtiene una media de $3,645\left(\sigma=1,763\right.$ y $\left.\sigma^{2}=3,110\right)$, lo que significa que los docentes "a veces" perciben a sus líderes con características transaccionales. Por su parte, para el estilo transformacional la situación no es distinta, ya que la media obtenida es de 3,725 ( $\sigma=1,836$ y $\left.\sigma^{2}=3,370\right)$, lo que indica que los líderes de ingeniería ocasionalmente muestran actitudes transformadoras. Por último, para el estilo pasivo/evitador se obtiene una media de 3,671 ( $\sigma=1,685$ y $\left.\sigma^{2}=2,838\right)$, indicando así que los líderes "a veces" presentan actitudes de este estilo.

Tabla 3: Estadísticos descriptivos para el liderazgo

\begin{tabular}{|l|c|c|c|}
\hline Estilo de liderazgo & Media aritmética & Desviación Típica & Varianza \\
\hline Transaccional & 3,645 & 1,763 & 3,110 \\
\hline Transformacional & 3,725 & 1,836 & 3,370 \\
\hline Pasivo/evitador & 3,671 & 1,685 & 2,838 \\
\hline
\end{tabular}

Ahora bien, en la Tabla 4 se observan los porcentajes de las respuestas obtenidas por cada dimensión del liderazgo. Estos resultados sustentan el análisis anterior, en sentido de que los máximos valores obtenidos radican en los puntajes medios (en ninguna dimensión los extremos tienen el mayor porcentaje, a excepción de "Recompensa contingente" que comparte el mismo puntaje entre "Nunca", "A veces" y "Bastante a menudo"). De igual manera, en 7 de las 9 dimensiones el mayor porcentaje se encuentra en el lugar 4, lo que sustenta las conductas ocasionales de estas características. Por último, en todas las dimensiones el porcentaje acumulado entre "Nunca", "Rara vez" y "De vez en cuando" es superior a "Bastante a menudo", "Con mucha frecuencia" y "Siempre", lo que indica que los docentes no perciben a sus directivos con ninguna dimensión en particular.

Tabla 4: Resumen de respuestas por dimensión (\%). Los números corresponden a: 1: "Nunca”; 2: "Rara vez"; 3: "De vez en cuando"; 4: "A veces"; 5: "Bastante a menudo"; 6: "Con mucha frecuencia"; 7: "Siempre"

\begin{tabular}{|c|c|c|c|c|c|c|c|c|}
\hline \multicolumn{2}{|r|}{ Estilos y dimensiones } & 1 & 2 & 3 & 4 & 5 & 6 & 7 \\
\hline \multirow{2}{*}{$\begin{array}{l}\text { Liderazgo } \\
\text { transaccional }\end{array}$} & Recompensa contingente & 16,79 & 14,60 & 12,41 & 16,79 & 16,79 & 13,87 & 8,76 \\
\hline & Administración por excepción activa & 15,11 & 12,95 & 17,27 & 28,78 & 14,39 & 10,07 & 1,44 \\
\hline \multirow{5}{*}{$\begin{array}{l}\text { Liderazgo } \\
\text { transformacional }\end{array}$} & Influencia idealizada conductual & 11,11 & 16,91 & 16,43 & 19,32 & 11,59 & 17,39 & 7,25 \\
\hline & Influencia idealizada atribuida & 18,29 & 17,71 & 9,71 & 20,57 & 10,29 & 16,00 & 7,43 \\
\hline & Motivación inspiracional & 15,79 & 18,18 & 16,27 & 16,75 & 14,35 & 13,40 & 5,26 \\
\hline & Estimulación intelectual & 10,63 & 21,74 & 14,01 & 16,91 & 15,46 & 9,18 & 3,38 \\
\hline & Consideración individualizada & 13,46 & 14,42 & 16,35 & 16,83 & 12,02 & 16,83 & 10,10 \\
\hline \multirow{2}{*}{ Pasivo/evitador } & Laissez-faire & 9,75 & 21,30 & 11,91 & 22,74 & 15,88 & 11,91 & 6,50 \\
\hline & Administración por excepción pasiva & 9,35 & 23,02 & 13,67 & 31,65 & 10,79 & 7,19 & 4,32 \\
\hline
\end{tabular}

Por otro lado, en base al análisis de la cultura organizacional, en la Figura 2 se puede observar a niveles generales la percepción que los docentes de ingeniería tienen sobre sus unidades académicas. En relación a los resultados, se puede decir que a pesar de que comparten rasgos de los cuatro tipos de culturas, existe una mayor dominancia de la cultura de mercado $(66,3 \%)$ por sobre la de clan $(60,94 \%)$, la jerárquica $(60,57 \%)$ y la adhocrática (58,04\%). Esto indica que en primer lugar, los docentes se identifican con una cultura basada en los resultados y la competencia, seguido de aquellas culturas donde se enfatizan los valores familiares y la buena actitud de sus miembros; el orden, la eficiencia y la estabilidad; y el emprendimiento y la creatividad, respectivamente. 


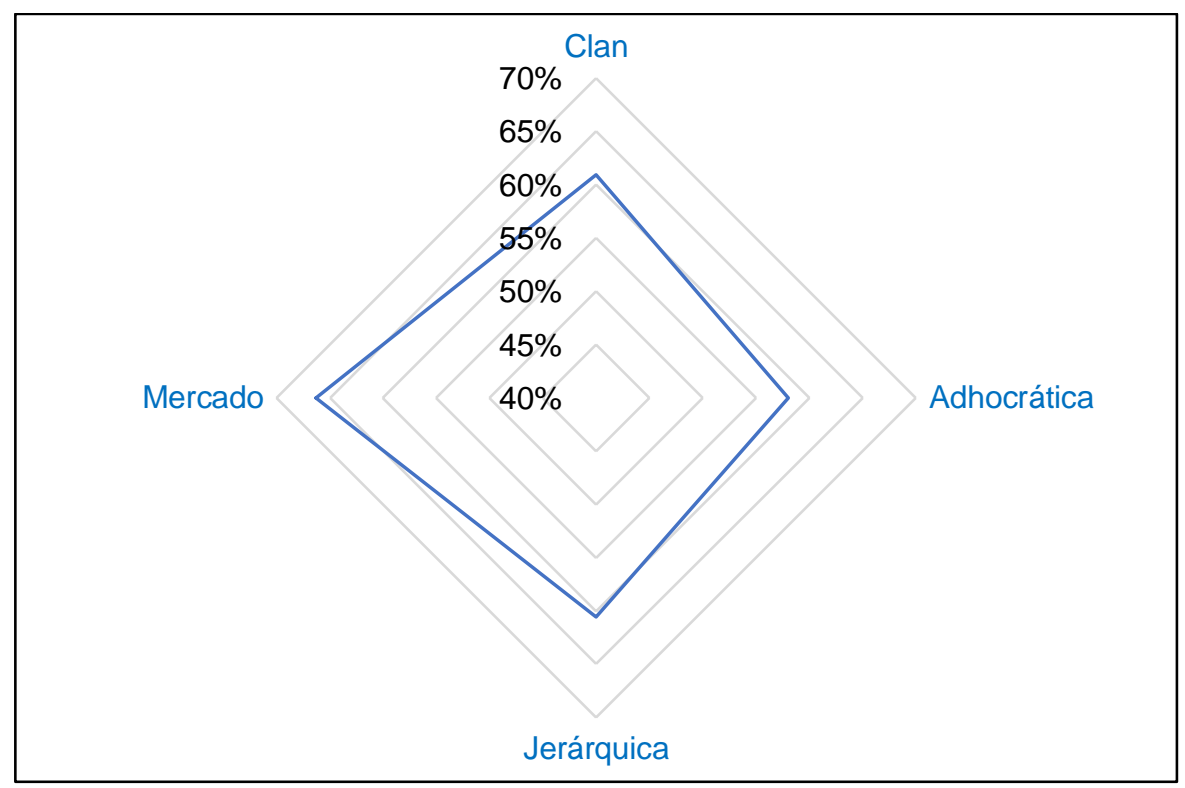

Fig. 2: Resultados del OCAI para las unidades académicas de ingeniería

En la Figura 3 se tienen los resultados obtenidos en la dimensión de características dominantes. Este indicador muestra que los docentes perciben a sus respectivas áreas como lugares muy controlados y estructurados y donde los procedimientos formales son muy importantes, resaltando de igual manera que tienden a ser áreas muy competitivas y orientadas a los resultados, lo cual corresponde a los estilos jerárquico $(66,5 \%)$ y de mercado $(63,6 \%)$, respectivamente. Más atrás le siguen las culturas de clan (58,0\%), relacionada con los valores familiares, y la adhocrática, en sentido de la promoción de la creatividad y la flexibilidad académica $(57,6 \%)$.

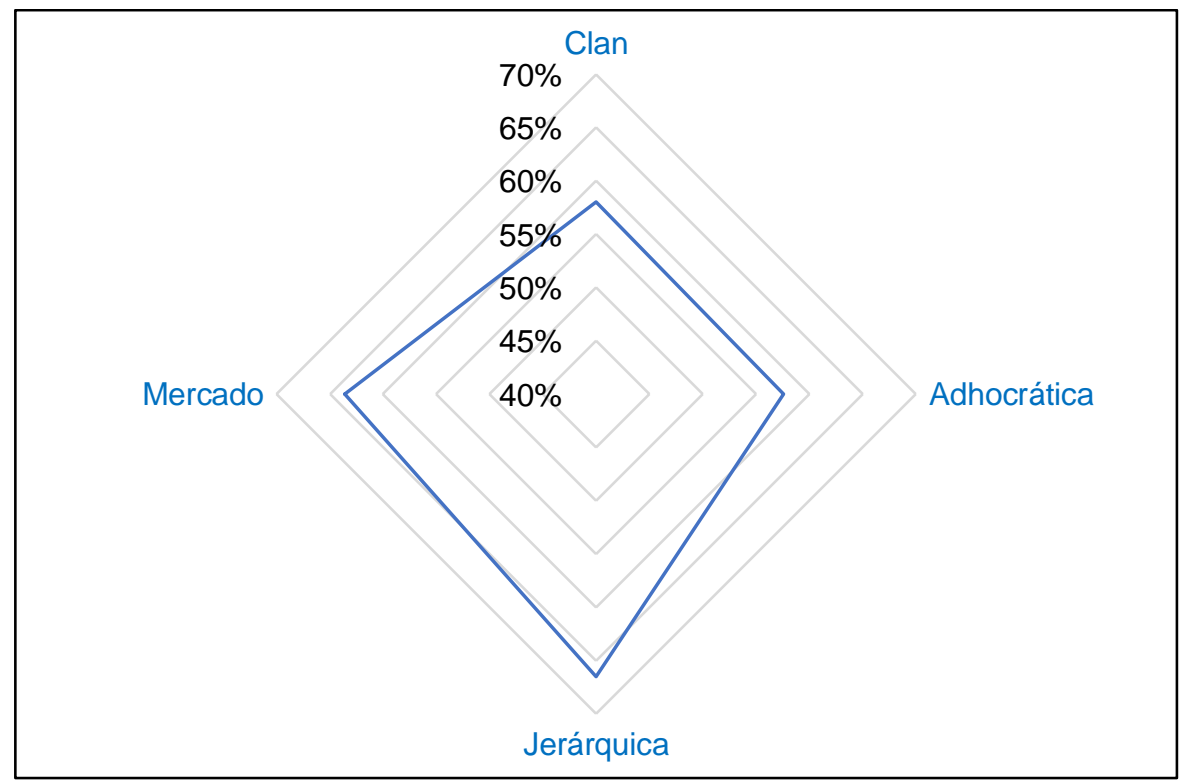

Fig. 3: Resultados del OCAI según dimensión de características dominantes

En la Figura 4 se muestra los resultados obtenidos en la dimensión de liderazgo organizacional. Este indicador muestra que existe una mayor tendencia a la cultura de mercado (67,0\%), lo que implica que los docentes tienden a pensar que sus líderes enfatizan y se orientan principalmente al logro de buenos resultados académicos. Más atrás, les siguen las culturas de clan $(62,1 \%)$, jerárquica $(59,4 \%)$ y adhocrática $(54,5 \%)$, en sentido de que estos perciben en menor medida a sus líderes como mentores que enfatizan la lealtad, como coordinadores de las actividades y los esfuerzos de manera racional, y como aquellos que estimulan la innovación docente. 


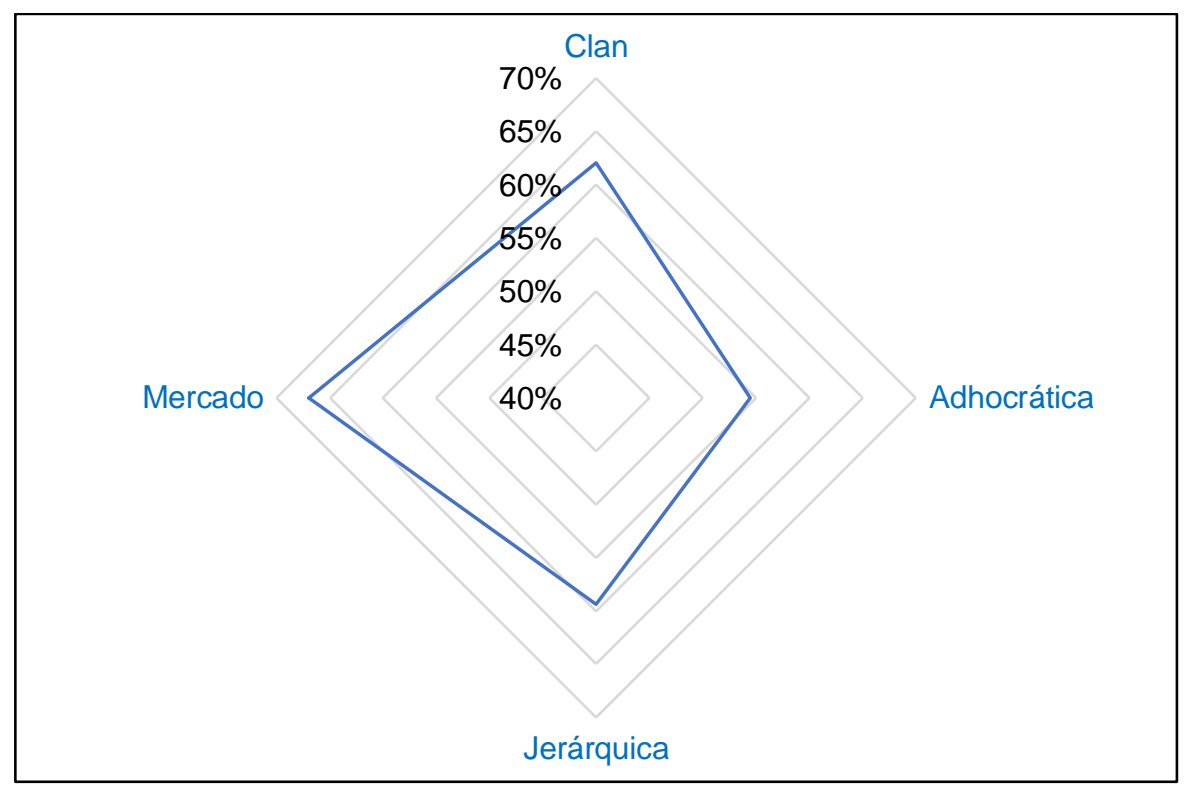

Fig. 4: Resultados del OCAl según dimensión de liderazgo organizacional

En la Figura 5 se puede observar los resultados obtenidos en la dimensión de gestión de empleados. Este indicador muestra que los docentes perciben que sus respectivas áreas tienden a caracterizarse por dar seguridad y estabilidad en los puestos de trabajo, por el incentivo de la participación y el trabajo en equipo, y por la alta exigencia en lo académico, características pertenecientes a los estilos jerárquico $(62,7 \%)$, de clan $(61,2 \%)$ y de mercado $(61,2 \%)$, respectivamente. En menor medida, creen que el estilo de gestión de sus respectivas unidades académicas se caracteriza por la toma de riesgo individual y la innovación, correspondiente al estilo adhocrático (57,1\%).

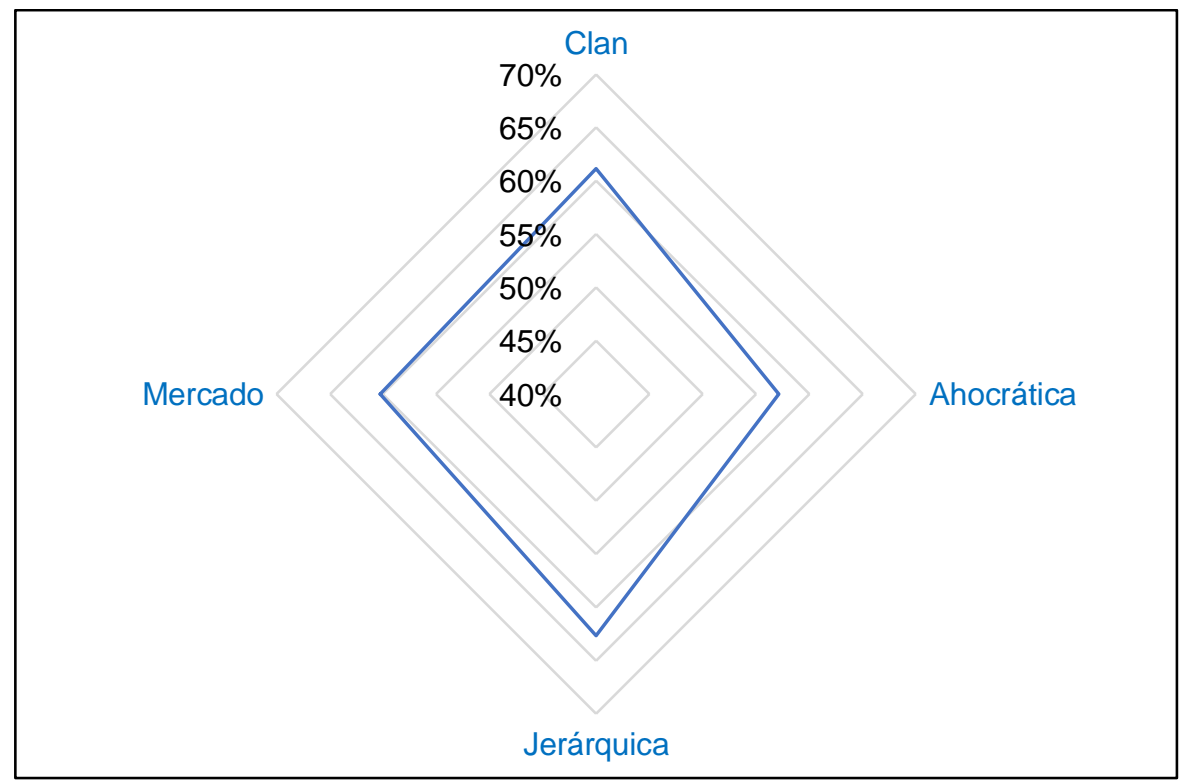

Fig. 5: Resultados del OCAl según dimensión de gestión de empleados

En la Figura 6 se muestran los resultados obtenidos en la dimensión de cohesión organizacional. Este indicador muestra que los docentes perciben que lo que en mayor medida mantiene unido a sus respectivas unidades académicas son las políticas y reglas existentes, seguido del cumplimiento de metas, y la lealtad y confianza entre sus miembros, correspondientes a los estilos jerárquico $(62,5 \%)$, de mercado $(61,2 \%)$ y de clan $(61,2 \%)$, respectivamente. En menor medida perciben que lo que los mantiene unidos son las características de la cultura adhocrática, relacionada con el compromiso en el crecimiento y la innovación académica $(54,9 \%)$. 


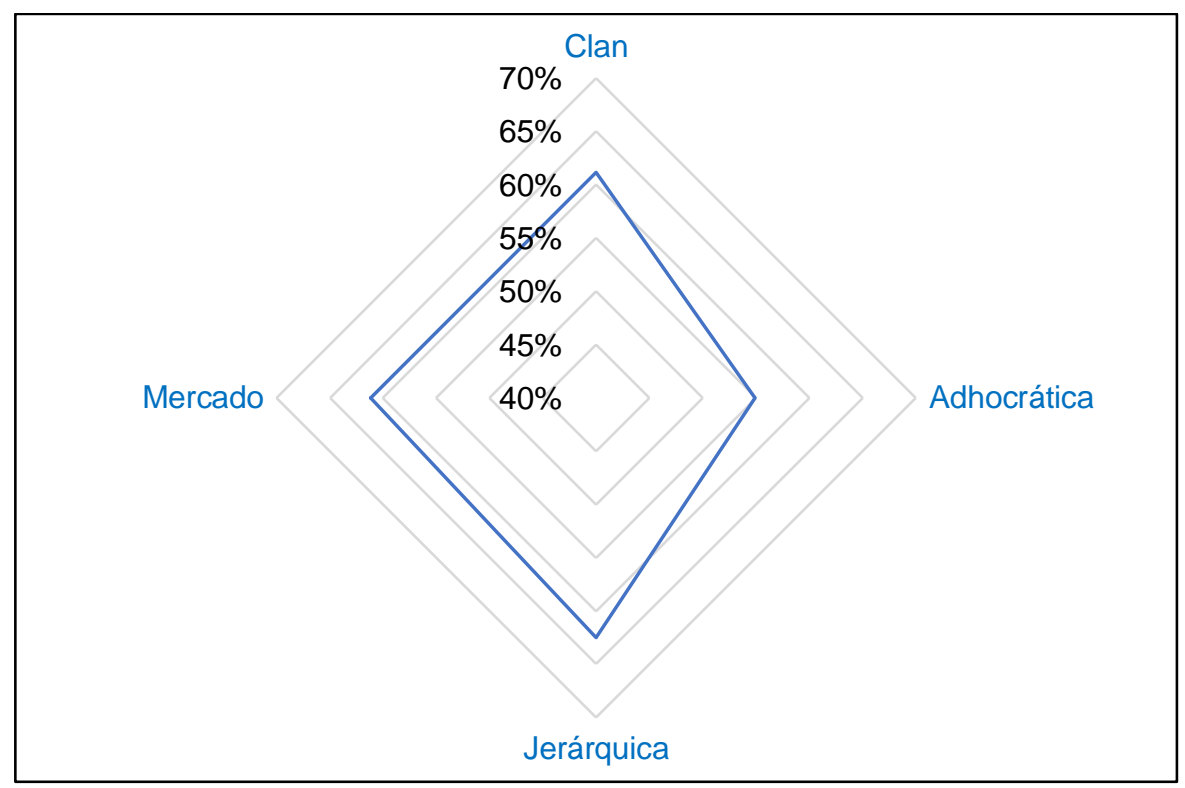

Fig. 6: Resultados del OCAl según dimensión de cohesión organizacional

En la Figura 7 se puede observar los resultados obtenidos en la dimensión de énfasis estratégico. Este indicador muestra que existe una mayor tendencia a las culturas adhocrática $(64,7 \%)$ y jerárquica $(64,7 \%)$, lo que implica que los docentes tienden a pensar que sus respectivas áreas de trabajo enfatizan la adquisición de nuevos recursos y desafíos, valorando asimismo la eficiencia, el control y la realización correcta del trabajo. Más atrás le siguen la cultura de clan, relacionada con el desarrollo y participación de los docentes $(59,4 \%)$ y la de mercado, vinculada a fomentar la competitividad $(58,9 \%)$.

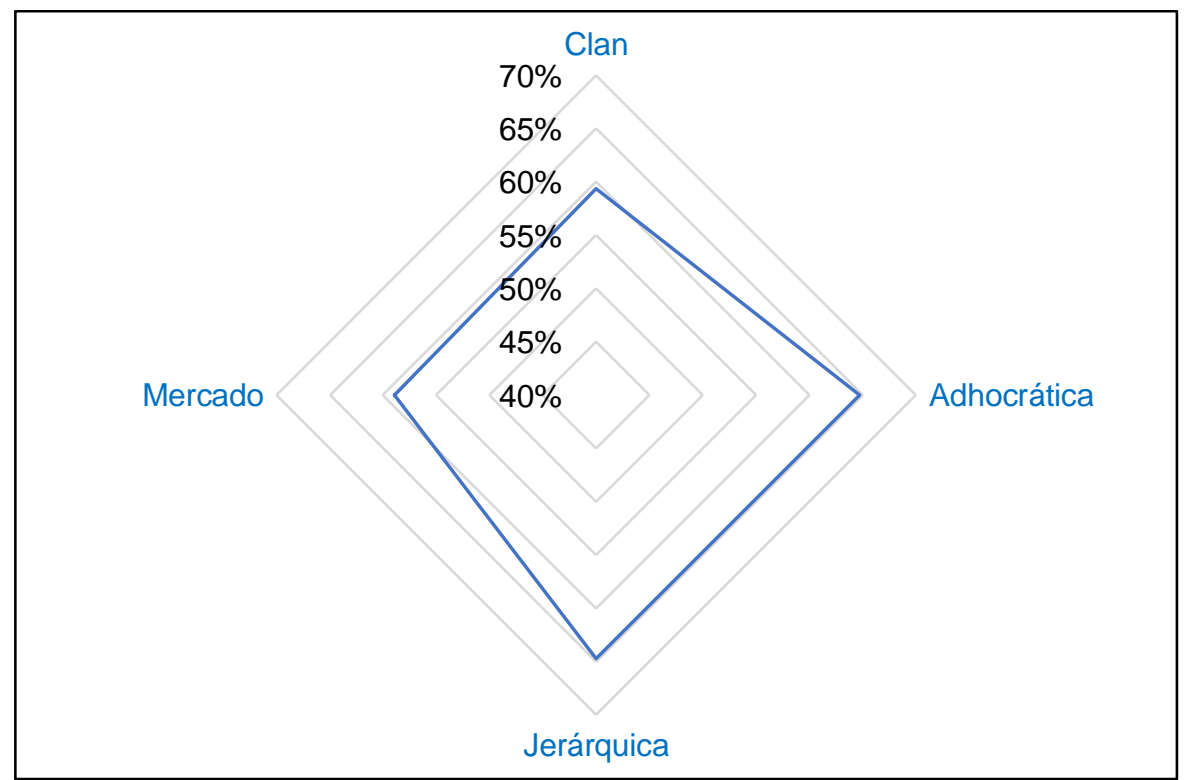

Fig. 7: Resultados del OCAI según dimensión de énfasis estratégico

Por último, en la Figura 8 se presentan los resultados obtenidos en la dimensión de criterios de éxito. Este indicador muestra que existe una mayor tendencia a la cultura jerárquica $(75,1 \%)$, lo que implica que los docentes tienden a pensar que el éxito de sus respectivas áreas se basa principalmente en el uso eficiente de recursos para el cumplimiento de sus tareas académicas. En menor medida, estos consideran que sus unidades definen el éxito sobre la base del desarrollo de los recursos humanos y de las relaciones, seguido por la superación frente a las demás áreas, y por ganar y alcanzar objetivos medibles, correspondientes a las culturas de clan $(62,5 \%)$, de mercado $(58,9 \%)$ y adhocrática $(57,6 \%)$, respectivamente. 


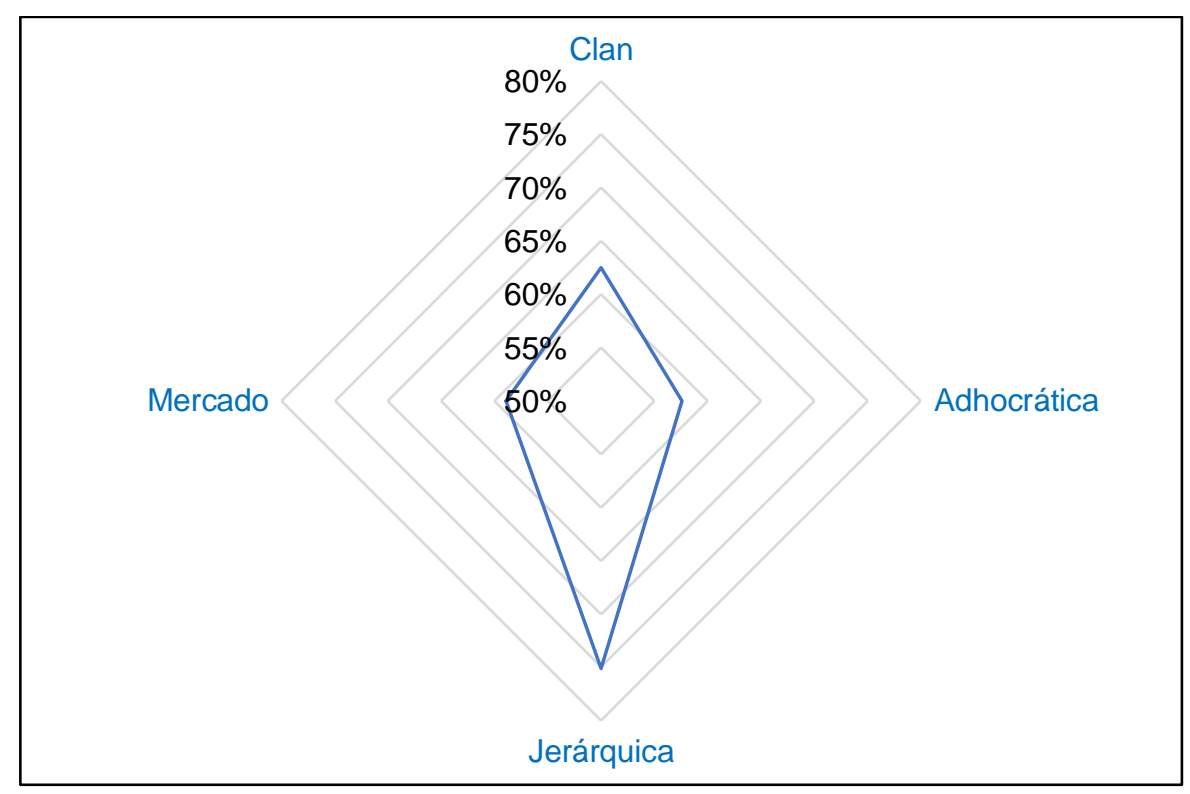

Fig. 8: Resultados del OCAI según dimensión de criterios de éxito

\section{RESULTADOS Y DISCUSIÓN}

Los resultados arrojaron que los directivos de ingeniería de la institución estudiada no tienen un liderazgo definido, sino más bien, presentan conductas ocasionales de los tres estilos, es decir, transformacional, transaccional y pasivo/evitador. Avolio y Bass (2004) plantean que dependiendo de las circunstancias en la que se encuentren, los líderes pueden combinar características transaccionales y transformacionales, ya que ellos definen el liderazgo como un proceso continuo entre estos dos estilos. Asimismo, en el ámbito educativo, autores como Hamilton (2010) sostienen que directivos que comparten características de estos liderazgos son más exitosos en la tarea de mejorar la ética docente así como el desempeño y la productividad académica y estudiantil, por lo que se considera que una mezcla efectiva de liderazgo transformacional y transaccional incorpora los elementos ideales para resolver los desafíos del presente y del futuro (Dartey-Baah, 2015). Por otro lado, autores como Al-Mansoori y Koç (2019), consideran a los líderes transformacionales como los más efectivos en cuanto a procesos universitarios, ya que estos son más eficaces al momento de aceptar el cambio y a desafiar el status quo, a diferencia de los líderes transaccionales quienes prefieren operar en ambientes rutinarios. En este contexto, otros estudios han posicionado al estilo transformacional como el más apto en el contexto educativo, como lo son por ejemplo Farrukh et al. (2019), quienes plantean que este liderazgo se asocia mejor con el comportamiento intraemprendedor (innovación, riesgo y proactividad) de los seguidores, asimismo Leal Filho et al. (2018) sostienen que este estilo puede fomentar la enseñanza y la educación para la sostenibilidad, mientras que Araneda-Guirriman et al. (2016) y Pedraja-Rejas et al. (2019) lo relacionan con mejores índices de calidad. A pesar de esta diferencia de opiniones (en relación a si es mejor ser un líder transformacional o una combinación de este liderazgo con el transaccional), varios autores coinciden en que el estilo pasivo/evitador es el más perjudicial en este contexto, ya que puede producir falta de motivación, colaboración y eficiencia en los seguidores (Suong et al., 2019). Es por esto que considerando los resultados obtenidos en esta investigación, sería interesante que los directivos buscaran formas de aumentar las conductas transformacionales y transaccionales, ya que estos estilos son percibidos como positivos en el contexto académico.

Por otro lado, para el caso de la cultura, la que resultó más predominante fue la de mercado, es decir, aquella basada en las tareas y los resultados, y la cual tiene un fuerte enfoque en la competencia, la flexibilidad, la movilidad y la productividad (Boichuk y Fast, 2017). En este sentido, diversos estudios demuestran que este tipo de cultura se correlaciona positivamente con la gestión de conocimiento (Chidambaranathan y Rani, 2015), la capacidad de aprendizaje organizacional (Dajani y Mohamad, 2016) y la calidad de las titulaciones universitarias (Pedraja-Rejas et al., 2018b). De igual manera, la cultura adhocrática, es decir, aquella que incentiva y pone como eje central la innovación y la creatividad de los seguidores, resultó con el menor porcentaje de percepción de los académicos, tanto a niveles generales como para cada una de sus dimensiones (exceptuando énfasis estratégico). Esto podría no ser bueno, ya que varios autores plantean que este tipo de cultura sería la más óptima en el contexto académico (González et al., 2016), ya que esta influye de manera positiva en los procesos de gestión de conocimiento (Adeinat y Abdulfatah, 2019), permite desarrollar un mejor desempeño a largo plazo (González et al., 2016), mejora la capacidad de respuesta antes situaciones emergentes y alienta a las personas a aprender cosas nuevas y tomar riesgos (Zhu y Engels, 2013). Por ende, se cree necesario que las unidades académicas de ingeniería de la universidad analizada 
deberían incentivar en mayor medida las conductas innovadoras de sus docentes, las cuales se pueden lograr al integrar tecnología interactiva y habilitando lugares para la visualización, la colaboración y la comunicación entre ellos (Eyal y Gil, 2020).

Finalmente, esta investigación es de tipo descriptiva, y como tal se limita a indagar la percepción de los docentes sobre el liderazgo y la cultura presente en las distintas unidades académicas de ingeniería de una universidad estatal chilena. De igual manera, posee una naturaleza exploratoria, lo que implica que "los resultados obtenidos no son susceptibles de ser empleados con fines de inferencia estadística" (PedrajaRejas et al., 2018b). Con todo, la investigación realizada puede servir como guía futura para profundizar los comportamientos en estos lugares y las posibles relaciones que estas tengan con otras variables de tipo organizacional.

\section{CONCLUSIONES}

Del análisis de la literatura, los resultados obtenidos y su posterior discusión, se extraen las siguientes conclusiones: 1) los instrumentos empleados en esta investigación cuentan con una sólida base teórica y empírica; 2) los directivos de ingeniería de la universidad estudiada no poseen un liderazgo definido, sino más bien presentan conductas ocasionales de los tres estilos planteados por Bass y Avolio, por lo cual se cree que estos deberían aumentar las conductas transformacionales y transaccionales ya que hay suficiente evidencia empírica para considerar a estos estilos como los más óptimos en el ámbito educativo; 3) la cultura predominante en las unidades académicas analizadas corresponde a la de mercado, mientras que la que menor percepción tuvo fue la adhocrática, es por esto que se plantea que las unidades deberían incentivar características propias de esta cultura como la innovación, la creatividad y la flexibilidad de los docentes, ya que esto podría resultar beneficioso a largo plazo; 4) una de las limitaciones de esta investigación es la naturaleza exploratoria y el trabajo a pequeña escala, lo cual entrega resultados no extrapolables bajo el enfoque estadístico; y 5) el estudio abre la oportunidad de llevar la investigación del liderazgo y la cultura organizacional a niveles de facultades y departamentos (ámbitos que son poco explorados aún) y a la posibilidad de realizar análisis de otra índole para determinar una posible vinculación con otras variables en el ámbito académico.

\section{AGRADECIMIENTO}

Los autores agradecen el patrocinio recibido por parte de ANID a través del Proyecto FONDECYT 1170960, titulado: La calidad en las instituciones de educación superior: la influencia de los estilos de liderazgo y la cultura académica. A su vez, se agradece el apoyo recibido a partir del proyecto PIA CIE 160007.

\section{REFERENCIAS}

Adeinat, I.M. y Abdulfatah, F.H., Organizational Culture and Knowledge Management Processes: Case Study in a Public University, https://doi.org/10.1108/vjikms-05-2018-0041, VINE Journal of Information and Knowledge Management Systems, 49(1), 35-53 (2019).

Al-Mansoori, R.S. y Koç, M., Toward Knowledge-Based Economy: Innovation and Transformational Leadership in Public Universities in Texas and Qatar, https://doi.org/10.3390/su11236721, Sustainability, 11(23), 1-30 (2019).

Araneda-Guirriman, C., Neumann-González, N., Pedraja-Rejas, L. y Rodríguez-Ponce, E., Análisis Exploratorio de las Percepciones sobre los Estilos de Liderazgo de los Directivos Universitarios en el Norte de Chile, http://dx.doi.org/10.4067/S0718-50062016000600013, Formación Universitaria, 9(6), 139-152 (2016).

Avolio, B.J. y Bass, B.M., Multifactor Leadership Questionnaire. Manual and Sampler Set, $3^{\mathrm{a}}$ ed., Mind Garden, California, USA (2004).

Bass, B.M. y Avolio, B.J., MLQ: Multifactor Leadership Questionnaire, Mind Garden, California, USA (2000).

Bass, B.M., Avolio, B.J., Jung, D.I. y Berson, Y., Predicting Unit Performance by Assessing Transformational and Transactional Leadership, https://doi.org/10.1037/0021-9010.88.2.207, Journal of Applied Psychology, 88(2), 207-218 (2003).

Berestova, A., Gayfullina, N. y Tikhomirov, S., Leadership and Functional Competence Development in Teachers: World Experience, https://doi.org/10.29333/iji.2020.13139a, International Journal of Instruction, 13(1), 607-622 (2020).

Boichuk, P.M. y Fast, O., Organizational Culture and Technology-Infused Management in Higher Education: Theoretical and Empirical Aspects, https://doi.org/10.33407/ittt.v61i5.1764, Information Technologies and Learning Tools, 61(5), 219232 (2017).

Bortolotti, T., Boscari, S. y Danese, P., Successful Lean Implementation: Organizational Culture and Soft Lean Practices, https://doi.org/10.1016/j.ijpe.2014.10.013, International Journal of Production Economics, 160, 182-201 (2015). 
Boulais, O., Torres, M. y otros cuatro autores, Developing Leadership and Global Professional Engineer Competences in our Students, https://doi.org/10.1109/RITA.2015.2452652, IEEE Revista Iberoamericana de Tecnologías del Aprendizaje, 10(3), 134-142 (2015).

Caliskan, A., y Zhu, C., Organizational Culture Type in Turkish Universities Using OCAl: Perceptions of Students, https://doi.org/10.15503/jecs20192.270.292, Journal of Education Culture and Society, 10(2), 270-292 (2019).

Cameron, K.S. y Quinn, R.E., Diagnosing and Changing Organizacional Culture: Based on the Competing Values Framework, $3^{a}$ ed., Jossey-Bass, San Francisco, USA (2011).

Cameron, K.S. y Quinn, R.E., Diagnosing and Changing Organizational Culture: Based on the Competing Values Framework, Revised ed., Jossey-Bass, San Francisco, USA (2006).

Carberry, A.R. y Baker, D.R., The Impact of Culture on Engineering and Engineering Education; in: Cognition, Metacognition, and Culture in STEM Education, pp 217-239. Springer, Cham (2018).

Cawn, B., Ikemoto, G., Grossman, J. y West, G., Ambitious Leadership: How Principals Lead Schools to College and Career Readiness, New Leaders, New York, USA (2016).

Chidambaranathan, K. y Rani, S., Knowledge Management and Organizational Culture in Higher Educational Libraries in Qatar: An Empirical Study, https://doi.org/10.1016/j.lisr.2015.11.002, Library \& Information Science Research, 37(4), 363369 (2015).

Dajani, M.A.Z. y Mohamad, M.S., Leadership Styles, Organisational Culture and Learning Organizational Capability in Education Industry: Evidence from Egypt, https://doi.org/10.18533/ijbsr.v6i11.1022, International Journal of Business and Social Research, 6(11), 42-57 (2016).

Daniëls, E., Hondeghem, A. y Dochy, F., A Review on Leadership and Leadership Development in Educational Settings, https://doi.org/10.1016/j.edurev.2019.02.003, Educational Research Review, 27, 110-125 (2019).

Dartey-Baah, K., Resilient Leadership: A Transformational-Transactional Leadership Mix, https://doi.org/10.1108/jgr-072014-0026, Journal of Global Responsibility, 6(1), 99-112 (2015).

Desselle, S., Rosenthal, M. y otros cuatro autores, Components of a Measure to Describe Organizational Culture in Academic Pharmacy, https://doi.org/10.5688/ajpe6022, American Journal of Pharmaceutical Education, 81(10), 6-19 (2017).

Elrehail, H., Emeagwali, O.E., Alsaad, A. y Alzghoul, A., The Impact of Transformational and Authentic Leadership on Innovation in Higher Education: The Contingent Role of Knowledge Sharing, https://doi.org/10.1016/j.tele.2017.09.018, Telematics and Informatics, 35(1), 55-67 (2018).

Englund, C., Olofsson, A. y Price, L., The Influence of Sociocultural and Structural Contexts in Academic Change and Development in Higher Education, https://doi.org/10.1007/s10734-018-0254-1, Higher Education, 76(6), 1051-1069 (2018).

Eyal, L. y Gil, E. Design Patterns for Teaching in Academic Settings in Future Learning Spaces, https://doi.org/10.1111/bjet.12923, British Journal of Educational Technology, 51(4), 1061-1077 (2020).

Farrukh, M., Lee, J.W.C. y Shahzad, I.A., Intrapreneurial Behavior in Higher Education Institutes of Pakistan: The Role of Leadership Styles and Psychological Empowerment, https://doi.org/10.1108/jarhe-05-2018-0084, Journal of Applied Research in Higher Education, 11(2), 273-294 (2019).

Fosnacht, K., Sarraf, S., Howe, E. y Peck, L.K. How Important are High Response Rates for College Surveys?, https://doi.org/10.1353/rhe.2017.0003, The Review of Higher Education, 40(2), 245-265 (2017).

Ganga-Contreras, F., Rodríguez-Ponce, E., Navarrete, E. y Pedraja-Rejas, L., Relevancia del Liderazgo en el Gobierno de las Universidades Iberoamericanas, Interciencia, 43(3), 160-167 (2018).

González, R., Ochoa, S. y Celaya, R., Cultura Organizacional y Desempeño en Instituciones de Educación Superior: Implicaciones en las Funciones Sustantivas de Formación, Investigación y Extensión, https://doi.org/10.12804/rev.univ.empresa.30.2016.01, Universidad \& Empresa, 18(30), 13-31 (2016).

Hamilton, M., The Interaction of Transactional and Transformational Leadership, Online Journal for Workforce Education and Development, III(3), 1-11 (2010).

Lai, C., Hsu, J. y Li, Y., Leadership, Regulatory Focus and Information Systems Development Project Team Performance, https://doi.org/10.1016/j.ijproman.2017.11.001, International Journal of Project Management, 36(3), 566-582 (2018).

Leal Filho, W., Raath, S. y otros ocho autores, The Role of Transformation in Learning and Education for Sustainability, https://doi.org/10.1016/j.jclepro.2018.07.017, Journal of Cleaner Production, 199, 286-295 (2018).

Lee, J., The Effects of Knowledge Sharing on Individual Creativity in Higher Education Institutions: Socio-Technical View, https://doi.org/10.3390/admsci8020021, Administrative Sciences, 8(2), 1-16 (2018).

Newell, C. y Bain, A., Academics' Perceptions of Collaboration in Higher Education Course Design, https://doi.org/10.1080/07294360.2019.1690431, Higher Education Research \& Development, 39(4), 748-763 (2019).

Pedraja-Rejas, L., Araneda-Guirriman, C., Bernasconi, A. y Viancos, P., Liderazgo, Cultura Académica y Calidad de las Universidades: Aproximación Conceptual y Relaciones, Revista Venezolana de Gerencia, 23(1), 184-199 (2018a). 
Pedraja-Rejas, L., Bernasconi, A. y otros cuatro autores, Cultura y Estilos de Liderazgo en Unidades Académicas: Un Estudio en una Institución de Educación Superior, Utopía y Praxis Latinoamericana, 24(Extra 4), $25-35$ (2019).

Pedraja-Rejas, L., Rodríguez-Ponce, E., Araneda-Guirriman, C. y Rodríguez-Ponce, J., La Cultura Organizativa en Unidades Académicas: Un Estudio Exploratorio desde Chile, Interciencia, 43(10), 729-734 (2018b).

Riquelme-Castañeda, J. y Pedraja-Rejas, L., El Liderazgo, la Cultura y los Desafíos de la Educación, http://dx.doi.org/10.4067/S0718-33052019000100005, Ingeniare: Revista Chilena de Ingeniería, 27(1), 5-7 (2019).

Rodzalan, S.A. y Saat, M.M., The Influence of Organizational Culture on Engineering Students' Team Working Skill Development, Proceedings of the Research in Engineering Education Symposium, 187-194 (2013).

Sainz-Martín, E., Igual-Catalán, R. y otros cuatro autores, Proposal to Apply a Code of Good Teaching Practice in Engineering, https://doi.org/10.1109/RITA.2017.2735518, IEEE Revista Iberoamericana de Tecnologías del Aprendizaje, 12(3), 116-123 (2017).

Schweisfurth, M., Davies L., Pe Symaco, L. y Valiente, O., Higher Education, Bridging Capital, and Developmental Leadership in the Philippines: Learning to be a Crossover Reformer, https://doi.org/10.1016/j.ijedudev.2017.09.001, International Journal of Educational Development, 59, 1-8 (2018).

Suong, H.T.T., Thanh, D.D. y Dao, T.T.X., The Impact of Leadership Styles on the Engagement of Cadres, Lecturers and Staff at Public Universities-Evidence from Vietnam, http://doi.org/10.13106/jafeb.2019.vol6.no1.273, Journal of Asian Finance, Economics and Business, 6(1), 273-280 (2019).

Taber, K.S., The Use of Cronbach's Alpha When Developing and Reporting Research Instruments in Science Education, https://doi.org/10.1007/s11165-016-9602-2, Research in Science Education, 48, 1273-1296 (2017).

Vasyakin, B., Ivleva, M. Pozharskaya, Y. y Shcherbakova, O., A Study of the Organizational Culture at a Higher Education Institution [Case Study: Plekhanov Russian University of Economics (PRUE)], International Journal of Environmental and Science Education, 11(10), 11515-11528 (2016).

Villa-Peralta, A., La Formación Educativa del Ingeniero y la Compleja Realidad del Mundo Contemporáneo, Aibi Revista de Investigación, Administración e Ingeniería, 7(2), 9-15 (2017).

Warrick, D.D., What Leaders Need to Know about Organizational Culture, https://doi.org/10.1016/j.bushor.2017.01.011, Business Horizons, 60(3), 395-404 (2017).

Wilson, D., Jones, D. y otros siete autores, Belonging and Academic Engagement among Undergraduate STEM Students: A Multi-Institutional Study, https://doi.org/10.1007/s11162-015-9367-x, Research in Higher Education, 56(7), 750-776 (2015).

Zhu, C. y Engels, N., Organizational Culture and Instructional Innovations in Higher Education, https://doi.org/10.1177/1741143213499253, Educational Management Administration \& Leadership, 42(1), 136-158 (2013). 